\title{
Atropine Induced Psychosis in Organophosphate Insecticide Poisoning and it's Management: A case series
}

\author{
Shoaib Asghar ${ }^{1}$, Junaid Mustafa ${ }^{1}$, Moeen Akhtar Malik ${ }^{1}$, Sohaib Asghar, ${ }^{1}$ Habib ur Rehman, ${ }^{1}$ Nida Gohar ${ }^{1}$
}

\begin{abstract}
Atropine is an established antidote in organophosphorus poisoning. It is an anticholinergic that inhibits the muscarinic actions of acetylcholine at postganglionic parasympathetic neuroeffector sites including secretary glands, smooth muscle, and CNS sites. In the literature, the cases of atropine intoxication are not uncommon. We report a case series of five cases of atropine induced psychotic disorder in patients, who manifested with delirium, hallucinations, nervousness, drowsiness, weakness, irrelevant talk, flushing along with tachycardia, and non-reactive dilated pupils suggesting possible anti-cholinergic abuse. The patients reported at the Department of Medicine-Unit II, Sheikh Zayed Medical College/Hospital, Rahim Yar Khan, from January to June 2019. The patients were managed symptomatically by titrating down the atropine's dose, administration of benzodiazepine, and antipsychotics.
\end{abstract}

Keywords: Organophosphorus poison, Atropine, Psychosis, Anti-cholinergic abuse

Article Citation: Asghar S, Mustafa J, Malik MA, Asghar S, Rehman Habib, Gohar N. Atropine Induced Psychosis in Organophosphate Insecticide Poisoning and it's Management: A case series. JSZMC 2021;12(02):23-25 https://doi.org/10.47883/jszmc.v12i02

This Open Access Article in Journal of Sheikh Zayed Medical College is licensed under a Creative Commons Attribution- 4.0 International License(CC BY 4.0).

\section{Introduction}

Organophosphate (OP) insecticide poisoning ${ }^{1,2}$ is the most common poisoning in developing countries due to ease of access. The acute nicotinic and muscarinic effects of organophosphate poisoning are well notified but neurobehavioral effects received less attention in the medical literature. There is a rise in reports of neuropsychiatric changes after both acute and chronic exposure. The crucial step in the case of organophosphate intoxications' management is the early identification of the symptoms and prompt diagnosis. The management includes skin decontamination, resuscitation with fluids administration, the muscarinic antagonist (atropine), and a cholinesterase reactivator (pralidoxime). The patient needs to be closely monitored for intermediate syndrome and recurrent cholinergic symptoms. The authors' present case series of five out of many clinical cases reported that developed atropine induced psychosis during treatment of organophosphate poisoning, its management, and outcome.

\section{Case Presentations}

There is an increased incidence of atropine induced psychosis in patients treated for acute organophosphate poisoning (OP). Patients were assessed initially for Muscarinic signs (salivation, lacrimation, urination, gastric upset, emesis, diaphoresis, diarrhea, meiosis, bradycardia, bronchospasm, bronchorrhea), Nicotinic Signs (tachycardia, weakness, fasciculation) and CNS signs of organophosphorus poisoning (headache, convulsions, drowsiness, unconsciousness).

As an initial phase in all cases, clinical ABCDE assessment was done and vitals were monitored. Primary care was given by gastric lavage with normal saline, skin decontamination by water body wash, and changing clothes. Continuous cardiac and vitals monitoring including oxygen saturation and blood glucose was performed. Supportive care was given using fluids. The patients were placed in the left lateral position to reduce the risk of aspiration, decrease absorption of the poison, and pyloric emptying. ${ }^{2}$ As maintenance phase, the patients were managed with intravenous (IV) cholinesterase reactivator- pralidoxime $1 \mathrm{~g} 8$ hourly and atropine repeatedly over 10-15 minutes as an anticholinergic agent at a dose of $2 \mathrm{ml} /$ hour until signs of atropinization.

Case 1: A 23-year-old male was admitted to the emergency department with sudden onset of

1.Department of Medicine, Sheikh Zayed Medical College/Hospital, Rahim Yar Khan, Pakistan.

Correspondence: Dr. Shoaib Asghar, Postgraduate Resident, Department of Medicine, Shaikh Zayed Medical College/Hospital, Rahim Yar Khan, Pakistan.

Email: dr.shoaibasghar@hotmail.com Received: 11-12-2019

Revised: $17-06-2021$ 
breathlessness, salivation, sweating, and multiple episodes of vomiting after suicidal ingestion of an unknown quantity of OP Poison 2 hours back. There was no history of seizures, altered sensorium delirium, psychosis, or paralysis. On examination, the patient was conscious, oriented with no focal deficits, and no neck muscle weakness. His vitals were: BP $110 / 80 \mathrm{mmHg}$, pulse: 89 beats/min, respiratory rate 20 breaths/minutes, SpO2: 98\%, blood sugar 125 $\mathrm{mg} / \mathrm{dl}$. On further examination, the patient had salivation, lacrimation, meiosis, tachycardia, gastric upset, vomiting, meiosis, and bronchorrhea. After 11 doses of atropine, the patient started experiencing agitation, delirium, visual and auditory hallucination, irrelevant talk, dry mouth, flushing, tachycardia, restlessness, and dilated pupils not reacting to light. ${ }^{3-6}$

All these symptoms post administration of atropine indicated anticholinergic drug-induced psychosis and hence a diagnosis of atropine psychosis, ${ }^{7,8}$ was considered. To manage this, atropine dose $\mathrm{e}^{2}$ was progressively reduced to $1 \mathrm{ml} / \mathrm{hr}, 0.5 \mathrm{ml} /$ hour, and then discontinued after signs of complete atropinization. The patient required symptomatic treatment, ${ }^{9,10}$ with benzodiazepine (IV diazepam $5-10 \mathrm{mg}$ ), and antipsychotics (IV haloperidol $5 \mathrm{mg}$ ) to manage agitation and other psychiatric symptoms.

Case 2: A 27-year-old pregnant ( 7 months) female admitted in an emergency after $150 \mathrm{ml}$ ingestion of OP one hour back. No muscarinic, nicotinic, or CNS signs of poisoning were present. Vitally stable. Gastric Lavage was done. Obstetric scan for fetal well-being was normal. After slow administration of 9 doses of IV atropine at 1 $\mathrm{ml}$ /hour, she developed atropine induced psychosis as confusion, drowsiness, and tachycardia. Treated by tapering off the dose of atropine and then stopped. Fetal and mother wellbeing monitored. No benzodiazepine or antipsychotics was given.

Case 3: A 32-year-old pregnant (4 months) mother of two admitted in an emergency after suicidal ingestion of OP along with her sister three hours back. Muscarinic signs of vomiting, diaphoresis, meiosis, bradycardia, and bronchospasm were present. After 14 doses of atropine at $2 \mathrm{ml} / \mathrm{hr}$, she developed atropine induced psychosis as agitation, confusion, and delirium. Treated with discontinuing atropine and administering IV benzodiazepine (diazepam) once. Fetal and mother wellbeing monitored by an obstetrician. Obstetric scan for fetal well-being was normal.

Case 4: A 18-year-old female admitted to emergency after ingestion of OP few hours back. Muscarinic and Nicotinic signs of poisoning were present. After 12 doses of atropine at $2 \mathrm{ml} /$ hour, she developed signs of atropine psychosis as irrelevant talk, agitation, nervousness, tachycardia, and hallucination. Treated with atropine withdrawal and symptomatic benzodiazepine (IV diazepam)

Case 5: A 24-year-old male admitted with severe shortness of breath after few hours of mixing and pouring insecticide (OP) in farm fields. Muscarinic signs of meiosis, vomiting, sweating, and bronchorrhea were present. After 14 doses of atropine at $2 \mathrm{ml} /$ hour, he developed signs of atropine psychosis as agitation, nervousness, and psychosis. Treated by stopping atropine, symptomatic benzodiazepine (IV diazepam and midazolam), and antipsychotic (IV haloperidol 5mg). These patients were discharged after few days in a much-improved condition. The causality assessment of psychosis with atropine using the Narajo ${ }^{6}$ causality assessment scale and WHO-Uppsala8 monitoring is indicated as probable association with atropine..$^{2,3,6}$ Thus, there is a temporal relation between the drug and the reaction.

\section{Discussion}

The atropine administration remains the mainstay for the pharmacological management of OP poisoning. The dose is adjusted for the individual patient accordingly so as to maintain the heart rate above 80 beats/minutes, systolic BP above $80 \mathrm{mmHg}$, and prevent the bronchorrhea or bronchospasm.

Toxic reaction ${ }^{2,4}$ to atropine results from its anticholinergic action and includes a variety of peripheral and central manifestations. This reaction is related to variation in the susceptibility to atropine, ${ }^{2}$ so that adverse reactions may occur at therapeutic doses that were not deemed excessive. Local signs of toxicity include dry skin, rash on face or chest, and conjunctival injection. Systemic toxicity causes tachypnea, temperature elevation, 
tachycardia, and CNS stimulation manifested as delirium, confusion, psychosis, restlessness, and occasionally seizures. In severe toxicity, central stimulation may cause respiratory or circulatory failure, CNS depression, coma, and death.

Adverse atropine reaction is managed with either discontinuing or continuing with some alteration. The discontinuation is done by withdrawal or replacement with others, whereas, continue the suspected drug, it may be dose reduced or replaced. Replacement of a drug can be done by physostigmine, scopolamine, and glycopyrrolate. ${ }^{2,10}$ Benzodiazepines and antipsychotics are used as drugs of choice in atropine psychosis. In a case like these, ${ }^{2,3,6}$ symptomatic treatment is appropriate, long-term therapy is not needed and intoxication is likely to resolve within days of discontinuing the offending agent.

\section{Conclusion}

Atropine induced psychosis is common in organophosphorus insecticide poisoning cases in adults. Early diagnosis and treatment may be lifesaving. Since atropine is one of the highly potent drugs used in the treatment of organophosphate intoxication, due care is essential while prescribing doses especially with IV administration and early withdrawal of the offending drug is crucial to avoid further complications due to central anticholinergic syndrome.

Authors Contribution: SA: Conception of work and Drafting. JM \& NG: Conception of work Interpretation of data and revising. MAM: Design of work and revising. SA \& HR: Analysis of data and drafting.

All authors critically revised and approve its final version.

Conflict of Interest: Authors has declared no conflict of interest.

Sources of Funding: The source of funding was self.

Disclaimer: None

\section{References}

1. Tom NR, Varghese GH, Alexander H, Swethalekshmi V, Ashok Kumar TR, Sivakumar T. A Case Report on Atropine Induced Psychosis. Int J Pharm Sci Res 2016; 7(1): 387-91.doi: 10.13040/IJPSR.0975-8232.7(1).387-91

2. Moudgil K, Tsundue T, Ponnusankar S. Atropine Induced Delirium in Organophosphate (OP) Insecticide Poisoning: A Case Report. J Young Pharm. 2018;10(2):2435.

3. Economacos G, Kanakis J. A case of hypersensitivity to atropine. Anesth Analg Reanim 1981; 38: 748.

4. Basha SA, Sathiswara B. Atropine induced psychosis: a report of two cases. Int J Health Sci Res. 2017; 7(12):325-327.

5. Organophosphrus poisoning. Available at; http://www.labome.org/research/Atropine-induceddelirium-which-developed-during-treatment-oforganophosphate-intoxication-in-adult-.html

6. Narajo scale. Available at: https://livertox.nih.gov/ Narajo.html

7. The use of the WHO-UMC system for standardised case causality assessment. Accessed from:https://www.who.int/medicines/areas/quality safety/safety_efficacy/WHOcausality_assessment. pdf

8. O'Connor PS, Mumma J: Atropine toxicity. Am J Ophthalmol 1985; 99: 613-14.

9. Eddleston M, Buckley NA, Eyer P, Dawson AH. Management of acute organophosphorus pesticide poisoning. Lancet.2008;371(9612):597-607.

10. Dogan ED, Gecici O. Central anticholinergic syndrome secondary to atropine treatment of organophosphate poisoning. Psychogeriatrics 2006;6:145-6. 